Medici and Francesco Sforza as both poisonous purveyors of private power is fodder for John M. Najemy's contribution. Carolyn James completes the volume with a telling essay showing that the Florentine dismissal of female authority stands in contrast to how signorial states empowered elite women. Capped with a robust bibliography, this collection updates the debate on the essential nature of Medicean rule, which will be vital for specialists and inviting for advanced students entering the debate.

JANICE LIEDL

Laurentian University

\title{
Butterworth, Emily.
}

\section{The Unbridled Tongue. Babble and Gossip in Renaissance France.}

Oxford : Oxford University Press, 2016. 233 p. et 7 ill. ISBN 978-0-19-966230-2 (relié) \$90.

Lorsqu'elle n'est pas tenue en bride, la langue, écrivent les théologiens moralistes, quitte rapidement le domaine de la vérité pour satisfaire inconsidérément son appétit de paroles, et de babillarde devient vaniteuse, sacrilège ou infamante. Après avoir consacré, il y a dix ans, un ouvrage à la diffamation en France à la Renaissance (Poisoned Words : Slander and Satire in Early Modern France, Londres : Legenda, 2006), Emily Butterworth se penche sur ce mal en amont qu'est l'incontinence verbale. Étroitement associés, les deux comportements langagiers demandent cependant des approches différentes : alors que la détraction se pense avant tout en termes légaux, le verbiage et le commérage, plus diffus, excèdent largement le cadre juridique. The Unbridled Tongue relève avec succès le défi que pose son objet multiforme en l'abordant à la fois sous un angle théologique, médical et légal. L'objectif est d'explorer les implications éthiques de la parole débridée et d'évaluer comment ses manifestations littéraires, souvent à forte teneur politique, témoignent des changements que connaissent les catégories du privé et du public aux $\mathrm{XVI}^{\mathrm{e}}$ et $\mathrm{XVII}{ }^{\mathrm{e}}$ siècles. Riche des réflexions suscitées dans le cadre du projet de recherche Gossip and Nonsense : Excessive Language in Renaissance France (2012-2015), dirigé par Hugh Gerald Roberts, cette monographie s'inscrit donc dans la lignée 
des travaux qui nuancent ou précisent le concept habermassien de "sphère publique bourgeoise » sous l'Ancien Régime.

La présentation de nombreux lieux communs tirés, pour l'essentiel, de la Lingua d'Érasme et du traité De garrulitate de Plutarque permet, au premier chapitre, de poser les enjeux rhétoriques liés à la loquacité, envisagée dans son rapport de proximité avec la copia. Glissante, cette vertu oratoire doit être recherchée avec habileté, sans quoi les discours dégénèrent à mesure qu'ils s'enflent. Ce risque est illustré à l'aide des Sérées de Guillaume Bouchet et des Après-dinées de Nicolas de Cholières, où l'idéal de sociabilité qui soustend la forme du banquet est mis à mal par des interventions trop copieuses. Se méfiant du pouvoir de prolifération anarchique du langage, les convives tentés de s'ériger en censeurs du babillage se taisent plutôt que d'envenimer le climat discursif. Le deuxième chapitre poursuit l'examen du double tranchant de l'abondance oratoire avec la figure du prophète : l'inspiration divinatrice engendre des discours qui surpassent l'entendement de l'énonciateur, lequel est dès lors susceptible d'être démonisé ou taxé de charlatanisme par ses détracteurs. Le rapprochement de divers commentaires sur la Pentecôte sert habilement de cadre pour penser le décalage - miraculeux ou suspect - entre les compétences linguistiques acquises et les performances spontanées extraordinaires.

S'ensuivent cinq chapitres qui abordent chacun une œuvre permettant d'éclairer un aspect social du bavardage ou de toute parole pléthorique. Chez Rabelais, la « voix publique » a un statut ambigu qui tend soit vers la parrhesia (le franc-parler), soit vers la prosopopeia (la parole masquée). L'Heptaméron de Marguerite de Navarre donne à entendre le "bruit commun " qui, paradoxalement, couvre et dévoile à la fois ce qui appartient à l'intimité. En relayant le qu'en-dira-t-on de façon critique, la petite société de devisants atteint à une certaine exemplarité, indépendante de la valeur relative des exempla colportés. Les Discours des misères de ce temps, ensuite, permettent d'observer la réversibilité du blâme des excès langagiers dans le contexte des guerres de Religion : dépeints par Ronsard comme des amuseurs publics verbeux, les prédicateurs protestants condamnent en retour l'idéologie jargonneuse du poète catholique. Alors que ce dernier réfléchit aux conditions de légitimité de toute prise de parole sur la place publique et aux conséquences de la licence réformée pour la cohésion sociale, Montaigne s'attarde plutôt à la réception de la rumeur populaire au niveau individuel et discute de la tendance de tout 
un chacun à la faire sienne en l'amplifiant. Chez Brantôme, enfin, les ouï-dire et les anecdotes retiennent l'attention en leur qualité de monnaie marchande, essentielle pour évoluer dans le milieu mondain des Dames galantes.

La lecture proposée de ces cinq auteurs est traversée par l'idée que la corruption de la copia se traduit par deux débordements. D’une part, le flot de paroles excède le contrôle de l'orateur et rejoint le discours dilué qui est prêté aux femmes, aux fous, aux démons et aux bonimenteurs, figures emblématiques de l'éloquence creuse. D’autre part, en se répandant hors du milieu d'où il provient, il acquiert une autonomie dont il peut tirer un poids politique. Ce double mouvement - contradictoire puisqu'il discrédite la prolixité en même temps qu'il lui confère sa force agissante - est au cœur de l'œuvre qui fait l'objet du chapitre final, une série d'opuscules réunis en 1623 sous le titre Recueil général des caquets de l'accouchée. La chambre d'une bourgeoise relevant de couches y est le théâtre de bavardages qu'un indiscret publie de façon anonyme, à l'insu des commères. À mi-chemin entre la conversation privée et l'échange public, entre l'oral et l'écrit, le féminin et le masculin, les Caquets sont un espace trouble où l'exercice personnel de l'Opinion, pour cacophonique et irraisonné qu'il soit, n'est cependant pas dépourvu d'efficacité et trouve une portée collective.

Si les discours renaissants sur la loquacité menacent souvent de tomber dans le travers qu'ils condamnent, l'étude d'Emily Butterworth, elle, demeure toujours claire et concise. L'attention minutieuse portée à la circulation, au fil des auteurs étudiés, des métaphores de la parole effrénée renforce l'unité de l'ouvrage. L'identification d'intertextes religieux récurrents, surtout, met utilement en lumière les prémisses communes aux œuvres du corpus. Aussi le développement moins succinct de certaines observations théologiques aurait-il pu servir avantageusement le propos, notamment à l'occasion d'une remarque sur les dangers d'épanchements lors de l'acte de confession (22) : la parole mesurée, calculatrice, est pourtant autrement plus préoccupante pour les directeurs de conscience. En fait, la conceptualisation rhétorique de la garrulitas comme copia défectueuse ou comme envers de la brevitas a pu, par moments, occulter le fait qu'elle est également, dans l'économie des péchés, le contre-pied parfois préférable du silence coupable (mala taciturnitas), un vice majeur pour ceux qui ont un devoir de parole (prophètes, prédicateurs, etc.), comme le rappellent Carla Casagrande et Silvana Vecchio dans leur étude classique sur les Péchés de la langue (Paris : Cerf, 1991). De telles considérations 
connexes découlent toutefois de la fécondité du propos, non d'une nécessité de l'argumentation qui, fluide et convaincante, se lit avec plaisir et grand profit.

CHRISTIAN VEILLEUX

Université McGill

Campana, Joseph and Scott Maisano, eds.

Renaissance Posthumanism.

New York: Fordham University Press, 2016. Pp. 335 + 35 b/w ill. ISBN 978-08232-6956-3 (paperback) US\$35.

This collection of eleven essays offers a balanced assessment of the intersection between Renaissance humanism and "posthumanism." The latter questions the assumptions of human exceptionalism, especially those founded on humanity's supposedly unique rationality, and instead posits a more fallible and fluid range of human characteristics, ones more closely aligned with those of other animal and plant species and even mineral elements. As the editors, Joseph Campana and Scott Maisano, argue in their introduction, posthumanists have often treated Renaissance humanism as a single and closed set of ideas, according to which humanity's special dignity elevates it above the rest of the world. Yet the essays demonstrate the ways that Renaissance humanists anticipated the very features about humanity that posthumanists would like to accentuate. In the introduction's second section, they study Paradise Lost as an example of the blurring of human and animal sensibilities, by applying to the poem Giorgio Agamben's critique of Martin Heidegger's sense of the "open." The introduction overall showcases the volume's strengths and weaknesses: while the contributions address the complexities of Renaissance and indeed premodern conceptions of humanity, they succumb at times to a lack of clarity about their theoretical presuppositions.

In the volume's first essay, Kenneth Gouwens presents a lucid, magisterial overview of the issues at hand. He takes to task posthumanists' often limited representations of humanism by tracing the ways Renaissance historians and humanists themselves identified diverse notions of humanity: they recognized not only humanity's sublimity, but also its depravity, whereby thinkers used animal comparisons to question human exceptionalism. Stephen Campbell 